\title{
Laboratory testing of LoRa modulation for CubeSat radio communications
}

\author{
Alexander Doroshkin ${ }^{1}$, Alexander Zadorozhny ${ }^{1, *}$, Oleg Kus ${ }^{2}$, Vitaliy Prokopyev ${ }^{1}$, and Yuri \\ Prokopyev $^{1}$ \\ ${ }^{1}$ Novosibirsk State University, 630090 Novosibirsk, Russia \\ ${ }^{2}$ OKB Fifth Generation Ltd., 630090 Novosibirsk, Russia
}

\begin{abstract}
We report the results of the feasibility study of using LoRa modulation for radio communications between CubeSat at low Earth orbit and ground station. The main goal of the study is to define how Doppler effect affects a LoRa radio link. Results of laboratory testing have shown high immunity of LoRa radio link to Doppler shift and a possibility to use LoRa modulation in CubeSat radio communications without any limitations.
\end{abstract}

\section{Introduction}

Various modifications of frequency or phase modulation are usually applied in the radio communication systems of the nanosatellites CubeSat. However, using more sophisticated modulation methods can significantly advance the efficiency of the radio link. One of such methods is the LoRa modulation [1-3] widely used in the networks of machine-to-machine (M2M) communications in the networks of IoT wireless sensors. This modulation method is based on the spread-spectrum technique where the data is encoded by a wide-band chirp signal in which the frequency linear increases or decreases with time.

A peculiarity of the Earth-satellite radio link is a high speed of the spacecraft passing over the ground station, which causes a strong Doppler shift of the frequency of the transmitted signal at the receiving point. The spread-spectrum technique used in the LoRa modulation has an inherent low sensitivity to the Doppler effect [2]. However, the LoRa modulation specification does not contain clear criteria of its applicability under real conditions of a quick movement of receiver relatively transmitter. It concerns especially conditions with the dynamic Doppler effect when the frequency shift changes rapidly with time. This takes a place in the Earth-satellite radio link. Therefore, it is yet unclear whether it is possible to use the LoRa modulation in radio link between a satellite and ground station. This paper presents the results of laboratory study of the immunity of a radio link with the LoRa modulation to the Doppler effect under the conditions corresponding to low Earth orbit CubeSat flying over the ground radio communication station.

\footnotetext{
*Corresponding author: zadorozh@phys.nsu.ru
} 


\section{Laboratory equipment for testing LoRa modulation}

Radio frequency vector signal generator NI PXIe-5672 by National Instruments and a LoRa transceiver based on the SX1278 chip by Semtech Corporation were used for testing and experimental studying the radio link using the LoRa modulation.

The NI PXIe-5672 vector signal generator is used for the synthesis of a RF signal with the LoRa modulation. In addition, the NI PXIe-5672 makes it possible to introduce various random and deterministic interference into the synthesized signal, including frequency distortions caused by the Doppler effect during the passing of the satellite over a ground station. The LoRa signal is synthesized by means of the software, which was developed for this research in the LabVIEW using the NI-RFSG software. In the experiments performed, the LoRa radio signal with a carrier frequency of $430 \mathrm{MHz}$ was synthesized.

The LoRa SX1278 standard transceiver is used during the laboratory experiments as a receiver and a demodulator. The LoRa radio signal emitted by the NI PXIe-5672 generator, being synthesized taking into account Doppler shift, is received, demodulated and analyzed by the receiver. The analysis of the quality of the received signal and the data packages contained therein are carried out using data from the internal registers of the receiver.

\section{Testing of LoRa radio link for immunity to Doppler shift}

In order to synthesize the high-frequency radio signal with the LoRa modulation propagating in a radio link between the satellite and the ground station, it is necessary to set the Doppler frequency shift and its variation in time while the satellite is flying over the station. Let the transmitter emits a radio signal with a frequency $F_{0}$. Due to the Doppler effect, the receiver will get a signal with frequency

$$
F=\frac{1}{1+\frac{v}{c} \cos \beta} \cdot F_{0}
$$

where $v$ is the satellite velocity, $c$ is the light speed, $\beta$ is the angle between the satellite velocity vector and the direction to the ground station. Then the relative Doppler frequency shift $\delta F$ is defined by:

$$
\delta F=\frac{F-F_{0}}{F_{0}}=\frac{1}{1+\frac{v}{c} \cdot \cos (\beta)}-1
$$

Let us consider the satellite moving in a circular orbit of height $H$ and ground station situated in a plain of the orbit (Figure 1).

Taking into account that the satellite velocity in the circular orbit $v=\sqrt{g \cdot R /(1+H / R)}$ where $R$ is the Earth's radius and $g$ is the gravitational acceleration at the Earth's surface, it is easy to obtain the following from (2) and Figure 1:

$$
\delta F=\frac{1}{1+\frac{1}{c} \cdot \sqrt{\frac{g \cdot R}{1+H / R}} \cdot \frac{\sin (\varphi)}{\sqrt{(1+H / R)^{2}-2 \cdot(1+H / R) \cdot \cos (\varphi)+1}}}-1
$$

where 


$$
\varphi=\frac{\sqrt{g / R}}{(1+H / R)^{3 / 2}} \cdot t
$$

Here, the time $t$ is assumed to equal zero when the satellite is at the zenith above the ground station.

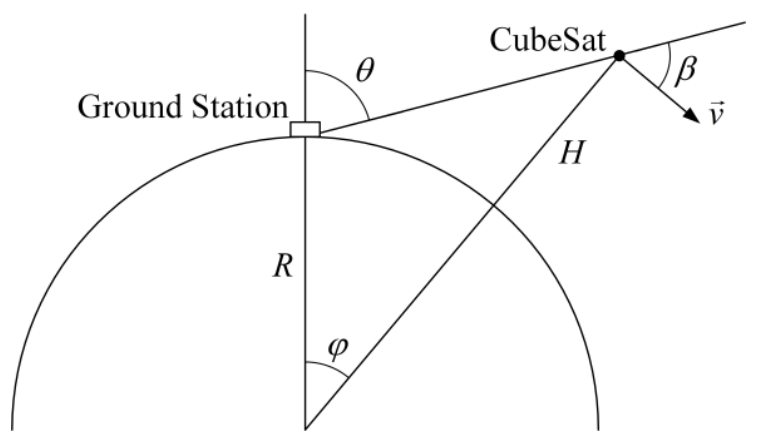

Fig. 1. The motion of the satellite in a circular orbit with respect to the ground station.

The Doppler effect is the most pronounced when the satellite moves at low altitudes, therefore its influence on the radio link with the LoRa modulation was investigated for the satellite moving in a circular orbit of $200 \mathrm{~km}$ high. The relative Doppler shift $\delta F$ in ppm units (parts per million) calculated by the formula (3) for this orbit and its time derivative are shown in Figure 2.

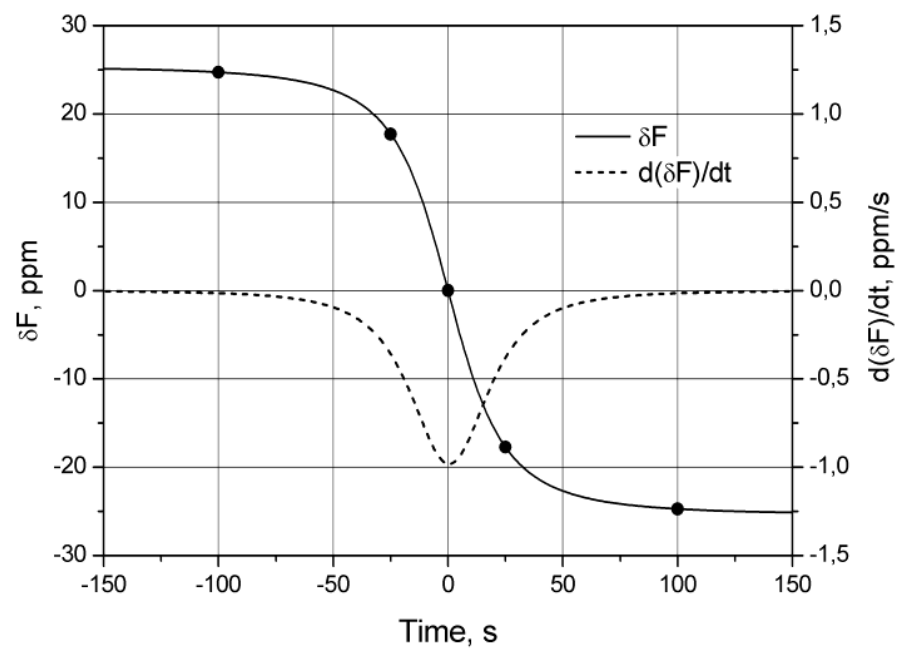

Fig.2. The relative Doppler shift $\delta F$ and its time derivative during the passing of the satellite at 200 $\mathrm{km}$ height over the ground station. The satellite is at the zenith at the time $t=0$. Bold points show times for which laboratory measurements were carried out.

In our experiments, the NI PXIe-5672 vector signal generator synthesized a RF LoRa signal with a frequency varying due to the Doppler effect in accordance with Figure 2 . That is the synthesized RF signal takes into account the dynamic Doppler Effect - the changing with time Doppler shift. The Doppler shift change reaches its maximum value when the satellite is at the zenith over the ground station. The synthesized RF signal was applied to 
the input of the SX1278 transceiver operating as a receiver and a demodulator. The criterion of communication quality was a $100 \%$ number of data received without loss from the entire package of the transmitted data. The data loss was detected via the check sum. The measurements were made at various LoRa modulation parameters and package durations.

The main parameters of the LoRa modulation, which determine the bit rate of the data signal, are the spreading factor $(S F)$ and the spread spectrum modulation bandwidth $(B W)$. The SX1278 transceiver using in the experiments offers $B W$ options ranging from $7.8 \mathrm{kHz}$ to $500 \mathrm{kHz}$ with $S F$ ranging from 6 to 12 . The immunity of the radio link with the LoRa modulation to the Doppler effect was tested with two sets of the parameters, which are given in Table 1. In the first set, the transmission time of a LoRa packet is close to the lowest possible in the standard LoRa for maximum payload size, and in the second set, it is close to the maximum possible one.

Table 1. LoRa modulation parameters used in testing LoRa radio link for immunity to Doppler effect.

\begin{tabular}{|c|c|c|c|c|}
\hline $\begin{array}{c}\text { No. } \\
\text { of set }\end{array}$ & $S F$ & $B W, \mathrm{ppm}$ & $\begin{array}{c}\text { LoRa payload size, } \\
\text { byte }\end{array}$ & $\begin{array}{c}\text { Transmission time of } \\
\text { a LoRa packet, } \mathrm{s}\end{array}$ \\
\hline 1 & 7 & 125 & 255 & 0.55 \\
\hline 2 & 11 & 250 & 255 & 2.5 \\
\hline
\end{tabular}

Bold points in Figure 2 show the most specific elements of satellite trajectory in the context of the radio link conditions covering all extreme situations. These situations are the largest static Doppler effect when the satellite is near the horizon, the largest dynamic Doppler effect when the satellite is at the zenith, and simultaneous high Doppler shift and high velocity of its changes when the satellite is in the intermediate position. Testing LoRa radio link was carried out for these satellite trajectory legs.

To determine the LoRa radio link immunity margin at each selected elements of satellite trajectory, the LoRa modulated radio link was tested for several $\delta F$ values and its derivative. First, tests were carried out at nominal values, which are calculated by the formula (3) and are shown in Figure 2. Then the Doppler shift was multiplied by the escalation factor $Q>1$, and the tests were repeated for various increasing values of $Q$ until the connection was lost. In such a manner, the maximum possible value $Q_{\max }$ characterizing the LoRa radio link immunity margin to the Doppler effect was determined.

Preliminary measurements showed that an increase in the RF signal level did not cause the increase in the number of successfully received packages unless the Doppler shift became more critical one, and the connection was lost. Therefore, the level of RF LoRa signal was chosen slightly above the receiver's sensitivity threshold in all the experiments. The results of the radio link tests with LoRa modulation parameters from Table 1 are given in Table 2. Here $\delta F_{\max }$ and $(d(\delta F) / d t)_{\max }$ are the maximum values of the relative Doppler shift and its time derivative under which the data transmitted without loss, $Q_{\max }$ is corresponding immunity margin to the Doppler effect.

Table 2. Results of testing LoRa radio link for immunity to Doppler effect.

\begin{tabular}{|c|c|c|c|c|}
\hline \multirow{2}{*}{$S F, B W$} & \multicolumn{2}{|c|}{ Static Doppler effect $(t=100 \mathrm{~s})$} & \multicolumn{2}{c|}{ Dynamic Doppler effect $(t=0 \mathrm{~s})$} \\
\cline { 2 - 5 } & $Q_{\max }$ & $\delta F_{\max }, \mathrm{ppm}$ & $Q_{\max }$ & $\begin{array}{c}(d(\delta F) / d t)_{\max }, \\
\mathrm{ppm} / \mathrm{s}\end{array}$ \\
\hline 7,125 & 2.8 & 70 & $>10$ & $>10$ \\
\hline 11,250 & 5.2 & 130 & 4 & 3.9 \\
\hline
\end{tabular}


One can see from Table 2 that the tested LoRa radio link has, in the worst case, the immunity margin to the Doppler effect $Q_{\max }=2.8$. That is, in the worst case, the Doppler effect can disrupt the LoRa radio link performance only when the Doppler shift is more than 2.8 times greater than the shift caused by the satellite moving in a circular orbit of 200 $\mathrm{km}$ high. It is important to note that the LoRa radio link is more immune to the dynamic Doppler effect in case of the first set of the LoRa modulation parameters $(S F=7, B W=125$ $\mathrm{kHz}$ ) that is in case of the shorter transmission time of a LoRa packet. Under longer transmission time of a LoRa packet $(S F=11, B W=250)$, the situation is reversed. In the case of the static Doppler effect, the measured values $\delta F_{\max }$ (70 and $130 \mathrm{ppm}$ ) coincide rather well with the specification of the LoRa SX1278 transceiver [4] in the part of the tolerated frequency offset between the transmitter and the receiver for the same modulation modes and the carrier frequency of $430 \mathrm{MHz}$ (73 and $100 \mathrm{ppm}$, respectively). As for the dynamic Doppler effect, it should be noted that the immunity of the LoRa radio link under the considered conditions is ensured by the SX1278 transceiver only in the mode which supported the symbol synchronization. The third bit of the RegModemConfig3 register activates this mode.

\section{Conclusion}

The performed laboratory study showed a high immunity of the radio link using the LoRa modulation to the Doppler effect under conditions of radio communication between satellite at low Earth orbit and ground station. The immunity margin to the Doppler effect under these conditions is better than 2.8. The impact on the LoRa radio link of both static and dynamic Doppler effect was investigated. It is obtained that the immunity to the dynamic Doppler effect is ensured only in the mode which supported the symbol synchronization. In general, the obtained results showed that the LoRa modulation could be used in low Earth orbit CubeSat radio communications without any limitations.

This work was supported by the Ministry of Education and Science of the Russian Federation: Project No. 14.575.21.0154, Project Identification No. RFMEFI57517X0154.

\section{References}

1. Fractional-N Synthesized Chirp Generator. United States Patent No.: US 7,791,415 B2, Date of Patent: Sep. 7 (2010)

2. LoRa ${ }^{\mathrm{TM}}$ Modulation Basics. Application None AN1200.22 (Semtech Corporation, 2015)

3. L. Vangelista, IEEE Signal Processing Letters, 24, 1818 (2017)

4. $S X 1276 / 77 / 78 / 79-137 \mathrm{MHz}$ to $1020 \mathrm{MHz}$ Low Power Long Range Transceiver. Datasheet, Rev. 5 (Semtech Corporation, 2016) 